\title{
Covid 19 and Ivermectin Prevention and Treatment Update
}

\section{Hirsch Roberto $\mathrm{R}^{1}$ and Carvallo Hector $\mathrm{E}^{2 *}$}

${ }^{1}$ Director Career of Specialist in Infectology, Htal Muniz, UBA, Former Prof. UBA. Head, Infectious Diseases Department, Htal. F. J. Muniz (CABA), Argentina

${ }^{2}$ Prof. Medicine UAI y U.M.; Former Prof. Medicine UBA.; Former Director Ezeiza Public Hospital, Argentina

*Corresponding author: Carvallo Hector E, Prof. Medicine U.A.I., U.M. Former Prof. Medicine U.B.A.; Former Director Ezeiza Public Hospital, Argentina, Email: hymcarvallo@hotmail.com

\section{Conceptual Paper}

Volume 4 Special Issue 1

Received Date: October 22, 2020

Published Date: November 17, 2020

DOI: $10.23880 /$ jidtm-16000S1-007

\section{Abstract}

The number of new COVID 19 contagions and related deaths is increasing worldwide. Our early protocols (NCT04425850 and NCT04425863) duly submitted and released through the National Library of Medicine (USA) have given unmatched profs of efficacy and safety, but still await their time to become widespread. In the meantime, we have simplified their use, and added some extra tools to combat this permanent peril. The results of our further investigations are summarized below.

Keywords: COVID19; Prophylaxis; Treatment; Update

\section{Conceptual Field}

From the first imported case of COVID 19 (from Milan, Italy, on March 3, 2020), until today (October 16), 966,000 infections and 25,723 deaths, have been registered in Argentina [1]. If we consider the under-registration that emerges from those not tested - and we are based on the fact that the level of testing in Argentina is one of the lowest in the world - we conclude that the REAL number of cases should be exceeding 4 million by far of people. Likewise, if we take into account the excess of deaths, deaths from COVID should be counted at about 50,000 [2]. Surely, we will never know exactly one or the other data. The truth is that - in recent times - the WHO has definitively ruled out some drugs that initially seemed promising, but that successive clinical studies ended up stoning. This is the case with remdesivir, the ritonavir/ lopinavir combination, hydroxychloroquine, and interferon [3]. Likewise, the trials carried out with convalescent plasma at the Hospital de Clínicas José de San Martín and the Hospital Italiano (both in CABA), as well as the multicenter one carried out by the Ministry of Health of the Province of Buenos Aires, yielded disappointing results [4-6]. There are still treatments with equine serum, monoclonal antibodies and ivermectin, which we have dedicated ourselves to, and which we will update below.

\section{Ivermectin in Prevention}

Ivermectin has been used both alone and associated with carrageenan- for the prophylaxis of the population groups most exposed to contagion, such as Health Agents $[7,8]$. Its administration alone or in combination, has demonstrated unobjectionable efficacy, greatly optimizing biosecurity measures and personal protection elements (which it does not replace). In this sense, there are certain updates: 1) the use of ivermectin as a preventive of COVID 19 should not be limited only to Health Agents, but should be extended to the Security Forces, to all Essential Personnel who must be transferred in mass media, to population groups in confinement and / or overcrowded conditions (geriatric, psychiatric, prison, orphanage, slums, etc.), their cohabitating contacts and people with comorbidities.

2) Ivermectin should be used at a rate of 200 micrograms per kilogram of weight, in a weekly dose, which will be repeated 
in the same period, up to 8 weeks. After these two months, the adipose tissue will have accumulated enough ivermectin for its protective effect to last for another four months [9]. Those four "extra" months can be covered with carrageenan, which may have been used since the beginning of prevention or just added in the ninth week, since its use can be prolonged indefinitely [10].

\section{Ivermectin in Treatment}

The I.D.E.A. Protocol has been replicated in several Provinces (Corrientes, Jujuy, Salta, Tucumán) and is being incorporated in many others (Misiones, Santa Fe, Chubut, etc.). Several amendments have been added, due to the results obtained and new concepts that have emerged since the completion and elevation of the protocols in June 2020 [11]. They are detailed below:

1) The doses are repeated weekly, as many weeks as necessary in each individual case, until the patient is free of disease and / or risk.

2) The weekly schedule can be shortened to every 5 days, if the patient's condition so requires.
3) Bromhexine has been associated with success, outside of the original protocol, since it adds a blocking factor on TMPRSS2 receptors that is not achieved with other medications [12].

4) Carrageenan can be added -also outside the original protocol- in order to reduce the dissemination by aerosols of patients, minimize endogenous reinfection, and the risk of the Health Personnel in charge of their care [13].

5) Once the patient is released, the immune response achieved through "natural active immunization" should be measured, which means having contracted the disease. This immunity is inconsistent at the humoral level, and there is still no infrastructure to massify the search for cellular immunity $[14,15]$. For this reason, we consider that if the patient has not elevated the specific IgG in a qualitatively/ quantitatively satisfactory way, he should continue with the prophylaxis scheme, once externalized. Below, we include a list of the international Trials dealing with Ivermectin that have been currently submitted to the National Library of Medicine (USA). In capital letters, we marked our Trials, which have already been completed and have results (NCT 04425850 and NCT 04425863).

\begin{tabular}{|c|}
\hline Title \\
\hline Ivermectin vs. Placebo for the Treatment of Patients With Mild to Moderate COVID-19 \\
\hline Safety and Efficacy of Ivermectin and Doxycycline in Treatment of Covid-19 \\
\hline Efficacy of Ivermectin in Adult Patients With Early Stages of COVID-19 \\
\hline Hydroxychloroquine and Ivermectin for the Treatment of COVID-19 Infection \\
\hline Effectiveness and Safety of Ivermectin for the Prevention of Covid-19 Infection in Colombian Health Personnel \\
\hline Efficacy and Safety of Ivermectin and Doxycycline in Combination or IVE Alone in Patients With COVID-19 Infection. \\
\hline Ivermectin in Adults With Severe COVID-19. \\
\hline Ivermectin Effect on SARS-CoV-2 Replication in Patients With COVID-19 \\
\hline Ivermectin and Nitazoxanide Combination Therapy for COVID-19 \\
\hline Ivermectin Nasal Spray for COVID19 Patients \\
\hline Ivermectin vs. Placebo for the Treatment of Patients With Mild to Moderate COVID-19 \\
\hline Clinical Trial of Ivermectin Plus Doxycycline for the Treatment of Confirmed Covid-19 Infection \\
\hline Outpatient Use of Ivermectin in COVID-19 \\
\hline Usefulness of Topic Ivermectin and Carrageenan to Prevent Contagion of Covid 19 \\
\hline Efficacy of Ivermectin in COVID-19 \\
\hline The Efficacy of Ivermectin and Nitazoxanide in COVID-19 Treatment \\
\hline Sars-CoV-2/COVID-19 Ivermectin Navarra-ISGlobal Trial \\
\hline Ivermectin and Doxycycine in COVID-19 Treatment \\
\hline Prophylactic Ivermectin in COVID-19 Contacts \\
\hline Max Ivermectin- COVID 19 Study Versus Standard of Care Treatment for COVID 19 Cases. A Pilot Study \\
\hline Ivermectin vs Combined Hydroxychloroquine and Antiretroviral Drugs (ART) Among Asymptomatic COVID-19 Infection \\
\hline Ivermectin-Azithromycin-Cholecalciferol (IvAzCol) Combination Therapy for COVID-19 \\
\hline
\end{tabular}


Journal of Infectious Diseases \& Travel Medicine

\begin{tabular}{|c|}
\hline A Comparative Study on Ivermectin and Hydroxychloroquine on the COVID19 Patients in Bangladesh \\
\hline Efficacy of Subcutaneous Ivermectin With or Without Zinc and Nigella Sativa in COVID-19 Patients \\
\hline Early Treatment With Ivermectin and LosarTAN for Cancer Patients With COVID-19 Infection \\
\hline Ivermectin, Aspirin, Dexamethasone And Enoxaparin as Treatment of Covid 19 \\
\hline A Preventive Treatment for Migrant Workers at High-risk of COVID-19 \\
\hline Novel Regimens in COVID-19 Treatment \\
\hline Novel Agents for Treatment of High-risk COVID-19 Positive Patients \\
\hline Comparative Study of Hydroxychloroquine and Ivermectin in COVID-19 Prophylaxis \\
\hline A Study to Compare the Efficacy and Safety of Different Doses of Ivermectin for COVID-19 \\
\hline Trial of Combination Therapy to Treat COVID-19 Infection \\
\hline Anti-Androgen Treatment for COVID-19 \\
\hline Comparative Study of Hydroxychloroquine and Ivermectin in COVID-19 Prophylaxis \\
\hline Early Treatment With Ivermectin for Patients with COVID-19 Infection \\
\hline Comparative study on new drugs against SARS COV 2 \\
\hline
\end{tabular}

\section{Ivermectin (IVM) Mechanisms of Action}

There are two mechanisms of action already described that explain IVM ways of preventing COVID 19 activity. The first one is outside host cells, by provoking ionophores along the viral nucleocapside, thus allowing $\mathrm{OH}$ compounds in. This deconstructs the virus structure.

The other mechanism is inside the host cell, preventing the virus to use the alpha and beta 1 importing as carriers to reach the cell nucleus.

\section{References}

1. Kontis V, Bennett JE, Robbie M Parks, Jonathan Pearson Stuttard, Rashid T, et al. (2020) Magnitude Demographics tnd Dynamics of The Effect Of The First Wave of The COVID-19 Pandemic on All-Cause Mortality in 21 Industrialized Countries. Nat Med.

2. Steven H Woolf, Derek A Chapman, Roy T Sabo, Daniel M Weinberger, Latoya Hill, et al. (2020) Excess Deaths From COVID-19 and Other Causes. JAMA 324(15): 1562564.

3. OMS (2020) Ensayo clinico "Solidaridad" sobre tratamientos contra la COVID-19.

4. La Provincia Publico El Primer Estudio Del Pais Sobre El Uso De Plasma, Jueves.

5. Hospital Italiano de Buenos Aires (2020) Estudio PlasmAr. 01 Octubre.

6. Htal.de Clinica Jose de San (2020) MartinPlasma de convalecientes como tratamiento para la infeccion por
SARS-CoV-2 informe Setiembre.

7. Carvallo Hecto, Hirsch Roberto, Fajardo Francisco, Ciruzzi Juan, Martin Mirta (2020) Ivermectina, Corticoides, Aspirina y Enoxaparina en el Tratamiento del COVID 19. Revista Educandonos, Archivos Argentinos de Dermatologia 7(2): 56-58

8. Carvallo Hector E, Hirsch Roberto R (2020) COVID 19 PROTOCOLO I.D.E.A. S.A.P. Noticias Metropolitanas XXXI Agosto 77: 20-23.

9. Fink DW, Porras AG (1989) Pharmacokinetics of Ivermectin in Animals and Humans. In: Campbell WC (ed) Ivermectin and Abamectin, Springer, New York, NY, pp: $113-130$

10. Ricardo Pena Silva, Stephen B Duffull, Andrew C Steer, Sandra X Jaramillo-Rincon, Amanda Gwee, et al. (2020) Pharmacokinetic considerations on the repurposing of ivermectin for treatment of COVID-19. Br J Clin Pharmacol.

11. Clinicaltrials.gov identifiers: NCT 04425850 y NCT 04425863.

12. Khalil Ansarin, Ramin Tolouian, Mohammadreza Ardalan, Ali Taghizadieh, Mojtaba Varshochi, et al. (2020) Effect of bromhexine on clinical outcomes and mortality in COVID-19 patients A randomized clinical trial. Bioimpacts 10(4): 209-215.

13. Nagle V, Gaikwad M, Pawar Y, Dasgupta S (2020) Marine Red Alga Porphyridium sp. as a Source of Sulfated Polysaccharides (SPs) for Combating Against COVID-19. 2020040168. 
14. Chuan Qin, Luoqi Zhou, Ziwei Hu, Shuoqi Zhang, Sheng Yang, et al. (2020) Dysregulation of Immune Response in Patients With Coronavirus 2019 (COVID-19) in Wuhan, China. Clin Infect Dis 71(15): pp 762-768.
15. Ling $\mathrm{Ni}$, Fang Ye, Meng-Li Cheng, Yu Feng, YongQiang Deng, et al. (2020) Detection of SARS-CoV-2Specific Humoral and Cellular Immunity in COVID-19 Convalescent Individuals. Immunity 52(16) :pp 971-977. 